\title{
Status of Slow Extraction of High Intensity Protons from Brookhaven's AGS*
}

\author{
K. Brown ${ }^{\dagger}$, L. Ahrens, J.M. Brennan, J.W. Glenn, T. Roser, T. Russo, N. Tsoupas, K. Smith, K. Zeno \\ AGS Dept., BNL, Upton, NY
}

\section{Abstract}

The Brookhaven AGS third integer resonant extraction system allows the AGS to provide high quality, high intensity $25.5 \mathrm{GeV} / \mathrm{c}$ proton beams simultaneously to four target stations and as many as 8 experiments. With the increasing intensities (over $7 \times 10^{13}$ protons/pulse) and associated longer spill periods ( 2.4 to 3 seconds long), we continue to run with low losses and high quality low modulation continuous current beams. We have an active program of high energy physics experiments, including the high precision measurement of the muons magnetic moment [1] and the discovery of the rare Kaon decay, $\mathrm{K}+\rightarrow \pi+\nu \bar{\nu}$ [2] This program is continuing into the future with the rare symmetry violating process experiments [3] currently being designed to operate at the AGS. In this paper we will present results from operation of high intensity slow extraction, the problems we encounter, and our solutions to those problems.

\section{INTRODUCTION}

The Brookhaven AGS Resonant extraction system and the beam transport and switchyard systems were designed in the pre-AGS Booster era, $[5,6,7]$ when the kinetic energy of the injected beam was $200 \mathrm{MeV}$ ( $50 \mathrm{MeV}$ even further back in time). In the post-Booster era, this energy is now approximately $1.6 \mathrm{GeV}$. For these two energies the ratio of $\beta \gamma$ is approximately 3.5. Therefore the maximum possible beam emittance is over 3.5 times larger for postBooster high intensity beams. Even though the emittance is larger, through careful measurements and simulations we have been able to find optics solutions that allow clean extraction and transport of these high intensity beams.

In addition to the larger beams, other factors have changed significantly since the design of the AGS SEB systems. The AGS now uses fast quadrupole magnets to jump the gamma transition point during acceleration. For minimal beam losses to occur during the $\gamma_{t r}$ jump the momentum spread of the beam has to be minimized. This puts constraints on how large the longitudinal emittance can be. This is due to the highly distorted dispersion function created by the fast quadrupoles, which defines the momentum aperture.[8]

Our experiences with extracting high intensity protons has been discussed previously in [4]. In this report we will

\footnotetext{
* Work performed under Contract Number DE-AC02-98CH10886 with the auspices of the US Department of Energy.

$\dagger$ Email: kbrown@bnl.gov
}

provide an update on our experiences since that report.

\section{SEB HIGH INTENSITY PERFORMANCE}

Basic performance parameters are summarized in table 1.

Table 1: AGS SEB Performance Parameters

\begin{tabular}{|l|c|c|}
\hline Parameter & Value & Units \\
\hline Momentum & 25.5 & GeV/c \\
Peak Intensity & 71.5 & $10^{12}$ proton/pulse \\
Extract. Eff. & $96-98$ & $\%$ \\
Transport Eff. & $90-95$ & $\%$ \\
Rep. Period & $4-8$ & second \\
Flattop Length & $2-6$ & second \\
Spill Length & $1.8-5.8$ & second \\
Working Point & $8.67 / 8.76$ & Tune $\left(\nu_{x}, \nu_{y}\right)$ \\
Chromaticity & $-2.3 / 0.2$ & Chrom. $\left(\xi_{x}, \xi_{y}\right)$ \\
\hline
\end{tabular}

\section{BEAM LOSS ISSUES}

The primary beam loss issues in the past had not been in the extraction process itself, but in the transport of the extracted beam to target stations. Through emittance measurements and simulations we were able to reduce beam losses in the primary transport to very tolerable levels. Back in the extraction systems we find the extraction efficiency drops at the highest intensities. Figure 1 shows the extraction inefficiency as a function of internal beam intensity. At around $5 \times 10^{13}$ the inefficiency makes a sudden jump up from just under $2 \%$ to about $2.5 \%$. We operate the AGS with the vertical chromaticity positive above $\gamma_{t r}$ but we are unable to operate the horizontal chromaticity positive since the current set of sextupoles are not strong enough. We have no direct evidence that the effect is a consequence of operating with a negative horizontal chromaticity. Since the increased beam loss is modest $(0.5 \%)$ we tolerate it. There is some cause for concern, though, since we are working towards even higher intensities in the future and at $1 \times 10^{14}$ this loss becomes significant.

\section{SPILL STRUCTURE}

In the FY98/99 SEB run we ran into a new problem; significant spill structure not associated with power supply ripple. In the FY02 run we observed this effect again. This spill structure was analyzed and found to be random kilohertz oscillations. We found no correlations between these 


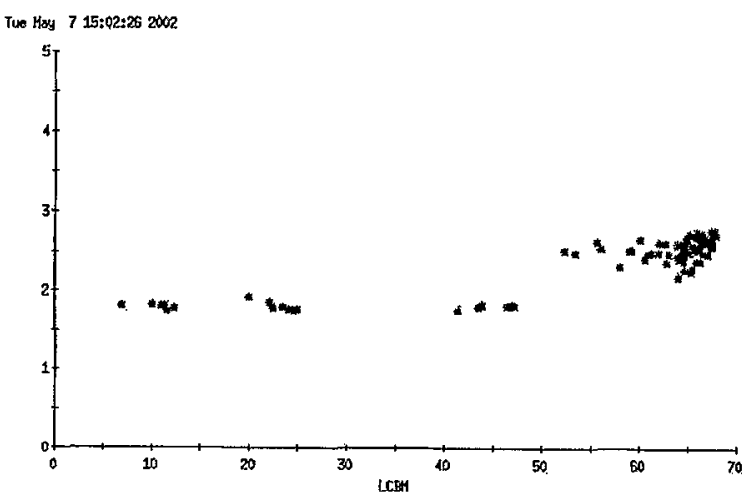

Figure 1: Extracted beam inefficiency vs internal beam intensity

oscillations and power supply ripples. The power supply ripple only accounted for about $20 \%$ of the spill structure. At high intensity the spill was $100 \%$ modulated (intensity dependent). Recall that the spill structure is a consequence of variations in velocities in tune space:

$$
S(t)=\frac{d N}{d Q} \cdot \dot{Q_{0}}\left(1+\frac{\dot{Q_{v}}}{\dot{Q}_{0}}\right)
$$

$\frac{d N}{d Q}$ is the distribution of particles in tune space. $\dot{Q}_{0}$ is the rate at which this distribution is pushed into the resonance. $\dot{Q}_{v}$ is the variations in the rate due to power supply ripple. When there is very little power supply ripple, the remaining structure is a consequence of the rate at which the beam is moved into resonance and the distribution of the particles in tune space. The random kilohertz structure appears to be a direct reflection of the distribution of particles in tune, or more properly, momentum space. Our solution to this problem is to use a $93 \mathrm{MHz} \mathrm{RF}$ (VHF) cavity during extraction, placing the $93 \mathrm{MHz}$ buckets between the beam and the resonance, such that the particles are forced between the RF buckets before going into non-linear resonant growth. Since we have a slight negative $\dot{B}$ during extraction the RF buckets will have only a small space between which the beam could pass, breaking up any structure that exists in the beam. This potentially puts $93 \mathrm{MHz}$ structure on the spill, which was not a problem for the experimenters using the beam. Figure 2 shows the beam spill with and without the VHF cavity on during extraction and at different intensities. Although the VHF cavity cures the effect, it is nevertheless unexplained. This phenomena is explored further in [10].

\section{ELECTROSTATIC SEPTUM DARK CURRENT}

One significant problem which effects our operation is the increasing dark current of the electrostatic septum over the period of a run. Figure 3 shows the septum power supply current as a function of integrated beam intensity during a portion of a physics run. Once the device is drawing the higher currents, it remains drawing those currents indefinitely. To return it to the lowest current levels has required

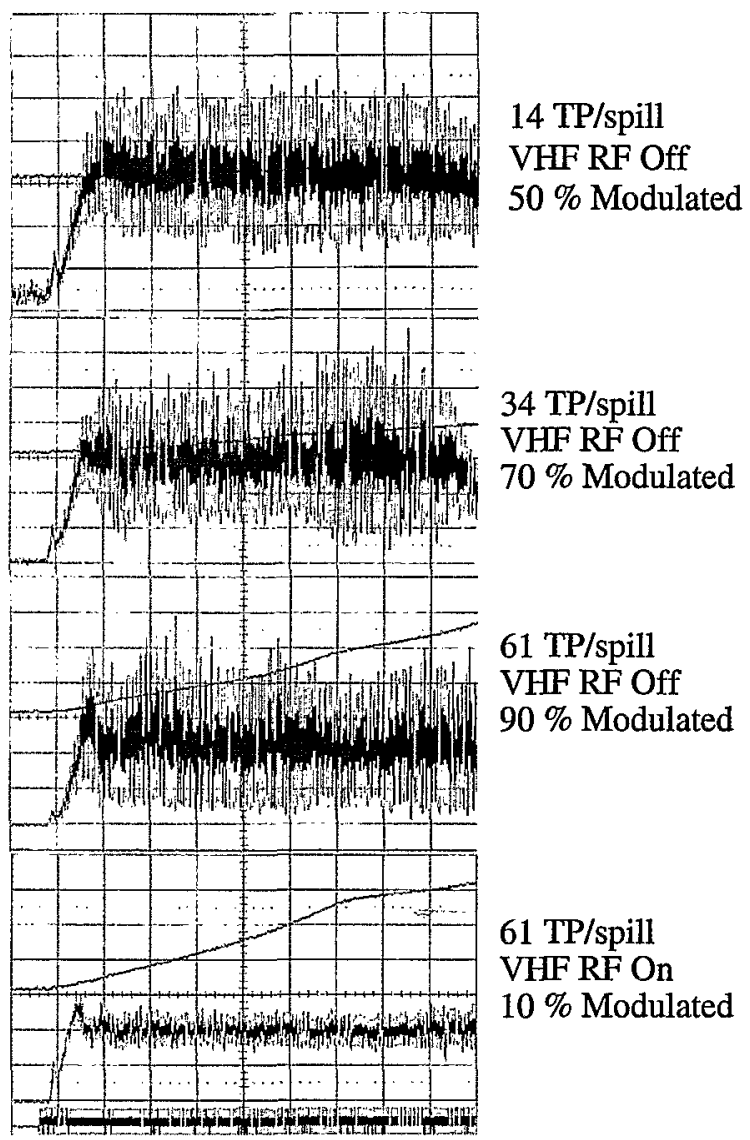

Figure 2: Extracted beam spill as a function of internal beam intensity with and without VHF on

the device to be brought back up to air and cleaned. Attempts to condition the device at higher voltages have not succeeded. This dark current effect has been observed in both our new foil septum and in the older wire septum. A detailed description of the new foil septum is found in [12].

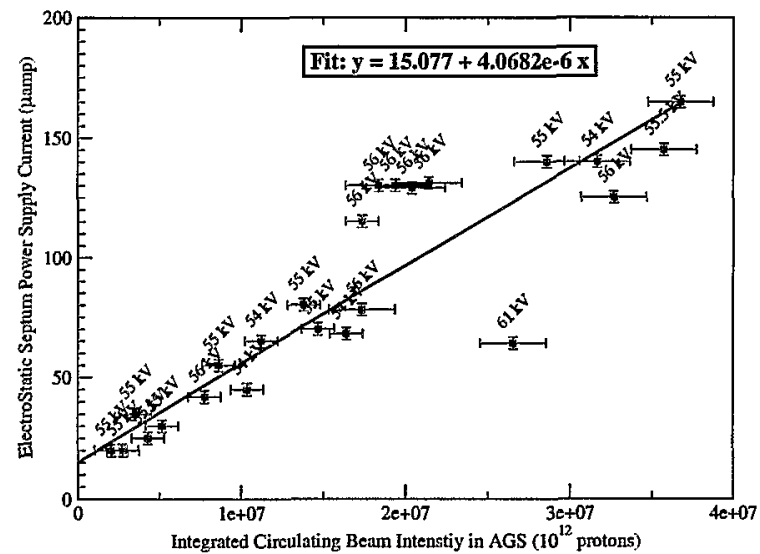

Figure 3: Electrostatic septum power supply current vs integrated beam intensity. Data labels indicate the high voltage as measured at the time. 


\section{CONCLUSIONS}

The AGS SEB system is able to supply high quality, high intensity proton beams for multiple simultaneous experiments. We are able to contend with instabilities that arise from the high current accelerated beams, as well as unexpected effects, such as spill structure uncorrelated with power supply ripple. For the AGS the VHF cavity has proven to be invaluable for diluting longitudinal phase space and now for smoothing spill structure.

\section{ACKNOWLEDGEMENTS}

The authors wish to acknowledge T. Curcio, who kindly collected and provided the data on the electrostatic septum power supply current.

\section{REFERENCES}

[1] H.N.Brown et al, "Precise measurement of the positive muon anomalous magnetic moment", Phys. Rev. Lett. 86, 2227 (2001)

[2] S.Adler et al,'Evidence for the Decay $\mathrm{K}+\rightarrow \pi+\nu \bar{\nu}$ ' Phys. Rev. Lett. 79, 2204-2207 (1997)

[3] Rare Symmetry Violating Processes Web Link, http://www.bnl.gov/rsvp/

[4] L.A.Ahrens et al, "AGS Resonant Extraction with High Intensity Beams", Proceedings of the 1999 Particle Accelerator Conference

[5] J.W.Glenn et al, "AGS Slow Extraction Improvements", IEEE NS26,No.3, 3167(1979)

[6] H. Weisberg and J.W. Glenn, "Exploitation of Nonlinear Growth of Betatron Oscillations to Obtain Efficient Slow Extraction at the AGS", Nucl. Inst. \& Methods 169, 319(1980).

[7] L. Blumberg et al, "The AGS Slow External Beam Switchyard", BNL 24508R (1979)

[8] L.A.Ahrens et al, "High Intensity Proton Acceleration at the Brookhaven AGS - An Update", Proceedings of the 1997 Particle Accelerator Conference, p.89, http://www.triumf.ca/pac97.html

[9] J.W. Glenn et al, "MICRO-BUNCHING THE AGS SLOW EXTERNAL BEAM", Proceedings of the 1997 Particle Accelerator Conference, p.967, http://www.triumf.ca/pac97.html

[10] J.W.Glenn et al, "Spill Structure in Intense Beams", These proceedings.

[11] Cappi \& Steinbach "Improvement of the Low Frequency ..." CERN/PS/OP 80-10 \& IEEE NS-28, No 3, June 1981, pp 2806

[12] J.Hock, “AGS Electrostatic Septum”, These Proceedings. 